\title{
Linear Quadratic Regulator for Impulse Uncontrollable Singular Systems
}

\section{Xiaoping Liu ${ }^{1,2 *}$ and Yufu Jia ${ }^{2}$}

${ }^{1}$ Department of Electrical Engineering, Lakehead University, Thunder Bay, ON, P7B 5E1, Canada

${ }^{2}$ Faculty of Electronics and Information Engineering, Liaoning University of Science and Technology, Anshan, Liaoning Province, China

\begin{abstract}
This paper investigates the linear quadratic regulator problem for singular systems which may contain some impulse uncontrollable modes. By transforming to the Weierstrass form, impulse controllable and uncontrollable subsystems can be separated. Sufficient conditions are proposed, under which solutions to both state and costate equations for the linear quadratic regulator problem are impulse-free. The necessary conditions for the minimization of the quadratic cost function are converted to a two-point boundary value problem, which can be solved by either computing its back-time state transition matrix or solving a Riccati equation. Two examples are provided to illustrate the main results.
\end{abstract}

Keywords: Singular systems; Descriptor systems; Linear quadratic regulator; Optimal feedback

\section{Introduction}

Singular systems, also called descriptor systems, arise naturally in chemical processes, mechanical systems, and so on. Therefore, since the last seventies, singular systems have attracted intensive attention. Linear quadratic regulator (LQR) problems play an important role in the field of control engineering. Therefore the LQR problems for descriptor systems have been investigated in the literature. The earliest papers on this problem are those of [1] and [2]. This problem were also addressed in ref. [3,4], where a generalized Riccati equation is shown to result directly from the necessary conditions for the existence of an optimal control. The generalized Riccati equation proposed in [3] may not have solutions, which was shown in ref. [5]. Bender and Laub [5] approached this problem by performing a singular value decomposition (SVD) to isolate the dynamics from non-dynamic portion, and four equivalent Riccati equations were derived in the SVD coordinate system. [6] investigated that the robust properties of the LQR for singular systems with single input. A parametrization of the optimal feedback gains was used to show that certain robust properties can be achieved by an appropriate choice of the feedback gain. The LQR problem for linear time-varying singular systems was addressed in ref. [7]. [8] derived a Riccati equation formulated in the original state parameters under which the problem has a solution. The LQR problem for non-regular singular systems was solved by converting a non-regular singular system into a regular one with index one in ref. [9].

It is worth pointing out that all the results on the LQR problem for singular systems mentioned above were obtained with an assumption that the singular systems are impulse controllable, that is, the impulsive modes can be removed by applying a state feedback. However, many practical systems, especially constrained mechanical systems (see Example 1), can not be modelled by an impulse controllable singular system. Therefore, the LQR problem for impulse uncontrollable singular systems is not only theoretically interesting but also practically important [10].

In this paper, the LQR problem for impulse uncontrollable singular systems is addressed. In order to guarantee the existence of the cost function, solutions to the singular systems should contain no impulse, which can be achieved by a feedback if the systems are impulse controllable or by choosing initial conditions properly for impulse uncontrollable cases [11]. Moreover, the final conditions should be properly chosen as well to guarantee that the optimal solution to the LQR problem is impulse-free. In order to facilitate the analysis, the singular system is assumed to be in the Weierstrass form [12].

\section{Problem Formulation}

Consider the problem of finding $u(t)$ to minimize

$$
J\left(x_{0}, u, t_{f}\right)=\frac{1}{2} x_{t_{f}}^{T} E^{T} Q_{f} E x_{t_{f}}+\frac{1}{2} \int_{0}^{t_{f}}\left[x^{T}(t) Q x(t)+u^{T}(t) R u(t)\right] d t
$$

with the constraint

$$
E \dot{x}(t)=A x(t)+B u(t), E x(0)=E x_{0}
$$

Where $R=R^{T}>0, Q=Q^{T} \geq 0, x(t) \in R^{n}, x(t) \in R^{n}, u(t) \in R^{m}$, and $\mathrm{E}$ is a square matrix of rank $q \leq n$. The pencil (sE-A) is assumed to be regular, that is, $\operatorname{det}(\lambda E-A) \neq 0$ for some $\lambda$. Let $\rho=\operatorname{deg}(\operatorname{det}(\lambda E-A))$. Assume that there exist invertible matrices $U$ and $V$ so that

$$
\begin{aligned}
& U E V=\left[\begin{array}{ll}
I & 0 \\
0 & N
\end{array}\right], U A V=\left[\begin{array}{cc}
A_{1} & 0 \\
0 & I
\end{array}\right], U B=\left[\begin{array}{l}
B_{1} \\
B_{2}
\end{array}\right], \\
& V^{-1} x=\left[\begin{array}{l}
x_{1} \\
x_{2}
\end{array}\right], U^{-T} p=\left[\begin{array}{l}
p_{1} \\
p_{2}
\end{array}\right], V^{T} Q V=\left[\begin{array}{ll}
Q_{11} & Q_{12} \\
Q_{21} & Q_{22}
\end{array}\right], \\
& U^{-T} Q_{f} U^{-1}=\left[\begin{array}{ll}
Q_{f 11} & Q_{f 12} \\
Q_{f 21} & Q_{f 22}
\end{array}\right], V^{-1} x_{0}=\left[\begin{array}{l}
x_{10} \\
x_{20}
\end{array}\right],
\end{aligned}
$$

where $p$ is the costate variable defined in (7) or (14), $x_{1}(t) \in R^{\rho}$, $x_{2}(t) \in R^{n-\rho}, \quad p_{2}(t) \in R^{n-\rho}, \quad p_{2}(t) \in R^{n-\rho}, \quad A_{1} \in R^{\rho \times \rho}, \quad B_{1} \in R^{\rho \times m}, \quad B_{2} \in R^{(n-\rho) \times m}$, $Q_{11}=Q_{11}^{T} \in R^{\rho \times \rho}, \quad Q_{12}=Q_{21}^{T} \in R^{\rho \times(n-\rho)}, \quad Q_{22}=Q_{22}^{T} \in R^{(n-\rho) \times(n-\rho)}, \quad Q_{f 11}=Q_{f 11}^{T} \in R^{\rho \times \rho}$, $Q_{f 12}=Q_{f 21}^{T} \in R^{\rho \times(n-\rho)}, Q_{f 22}=Q_{f 22}^{T} \in R^{(n-\rho) \times(n-\rho)}, N \in R^{(n-\rho) \times(n-\rho)}$ is a nilpotent matrix of the nilpotency $v$. Without loss of generality, assume that $\mathrm{N}$ takes a Jordan normal form of $\mathrm{N}=\operatorname{diag}\left\{N_{1}, \ldots, N_{\gamma}\right\}$ with the Jordon block $N_{i}$ being a shift matrix of size $v_{i} \times v_{i}$ and $v \geq v_{1} \geq \cdots \geq v_{\gamma_{1}} \geq v_{\gamma_{1}+1} \geq \cdots \geq v_{\gamma_{2}}>v_{\gamma_{2}+1}=\cdots=v_{\gamma}=1$, where $N_{i}=\left[\begin{array}{cc}0 & I_{v_{i}-1} \\ 0 & 0\end{array}\right] \in R^{v_{i}, v_{i}}$ with $I_{j}$ being a $j \times j$ identity matrix. Note that the first $\lambda_{2}$ Jordon blocks are nontrivial with the nilpotency greater than 1 and the rest are trivial with the nilpotency equal 1 .

${ }^{*}$ Corresponding author: Xiaoping Liu, Department of Electrical Engineering Lakehead University, Thunder Bay, ON, P7B 5E1 Canada, Tel: 807-343-8613; E-mail: xliu2@lakeheadu.ca

Received November 08, 2016; Accepted November 28, 2016; Published December 05, 2016

Citation: Liu X, Jia Y (2016) Linear Quadratic Regulator for Impulse Uncontrollable Singular Systems. J Electr Electron Syst 5: 207. doi:10.4172/2332-0796.1000207

Copyright: (c) 2016 Liu X, et al. This is an open-access article distributed under the terms of the Creative Commons Attribution License, which permits unrestricted use, distribution, and reproduction in any medium, provided the original author and source are credited. 
It will prove useful to partition $x_{2}, p_{2}, B_{2}, Q_{21}, Q_{22}, Q_{f 21}, Q_{f 22}$ as follows:

$$
\begin{aligned}
& x_{2}=\left[\begin{array}{c}
x_{2}^{1} \\
\vdots \\
x_{2}^{\gamma}
\end{array}\right], x_{20}=\left[\begin{array}{c}
x_{20}^{1} \\
\vdots \\
x_{20}^{\gamma}
\end{array}\right], p_{2}=\left[\begin{array}{c}
p_{2}^{1} \\
\vdots \\
p_{2}^{\gamma}
\end{array}\right], B_{2}=\left[\begin{array}{c}
B_{2}^{1} \\
\vdots \\
B_{2}^{\gamma}
\end{array}\right], Q_{21}=\left[\begin{array}{c}
Q_{21}^{1} \\
\vdots \\
Q_{21}^{\gamma}
\end{array}\right], Q_{22}=\left[\begin{array}{ccc}
Q_{22}^{11} & \cdots & Q_{22}^{1 \gamma} \\
\vdots & \ddots & \vdots \\
Q_{22}^{\gamma 1} & \cdots & Q_{22}^{\gamma}
\end{array}\right], \\
& Q_{f 21}=\left[\begin{array}{c}
Q_{f 21}^{1} \\
\vdots \\
Q_{f 21}^{\gamma}
\end{array}\right], Q_{f 22}=\left[\begin{array}{ccc}
Q_{f 22}^{11} & \cdots & Q_{f 22}^{1 \gamma} \\
\vdots & \ddots & \vdots \\
Q_{f 22}^{\gamma 1} & \cdots & Q_{f 22}^{\gamma \gamma}
\end{array}\right],
\end{aligned}
$$

with $\quad x_{2}^{i} \in R^{v_{i}}, \quad p_{2}^{i} \in R^{v_{i}}, \quad B_{2}^{i} \in R^{v_{i} \times m}, \quad Q_{21}^{i} \in R^{v_{i} \times \rho}, \quad Q_{22}^{i j}=\left(Q_{22}^{i j}\right)^{T} \in R^{v_{i} \times v_{j}}$, $Q_{f 21}^{i} \in R^{v_{i} \times \rho}, Q_{f 22}^{i j}=\left(Q_{f 22}^{j i}\right)^{T} \in R^{v_{i} \times v_{j}}$ for $i, j=1, \ldots, \gamma$.

Furthermore, for $i=1, \ldots, \gamma_{1}$, partition $x_{2}^{i}, p_{2}^{i}, B_{2}^{i}, Q_{21}^{i}, Q_{21}^{i}$, $Q_{22}^{i j}, \quad Q_{f 22}^{i j} \quad$ into $1 p t x_{2}^{i}=\left[\begin{array}{c}\hat{x}_{2}^{i} \\ \bar{x}_{2}^{i}\end{array}\right], \quad B_{2}^{i}=\left[\begin{array}{c}\bar{B}_{2}^{i} \\ \hat{B}_{2}^{i}\end{array}\right], \quad B_{2}^{i}=\left[\begin{array}{c}\bar{B}_{2}^{i} \\ \hat{B}_{2}^{i}\end{array}\right], \quad Q_{21}^{i}=\left(Q_{12}^{i}\right)^{T}=\left[\begin{array}{c}\hat{Q}_{21}^{i} \\ \bar{Q}_{21}^{i}\end{array}\right]$, $Q_{f 21}^{i}=\left(Q_{f 12}^{i}\right)^{T}=\left[\begin{array}{l}\hat{Q}_{21}^{i} \\ \bar{Q}_{21}^{i}\end{array}\right] Q_{f 21}^{i}=\left(Q_{f 12}^{i}\right)^{T}=\left[\begin{array}{c}\bar{Q}_{f 21}^{i} \\ \hat{Q}_{f 21}^{i}\end{array}\right], Q_{22}^{i j}=\left[\begin{array}{ll}\hat{Q}_{22}^{i j} & \bar{Q}_{22}^{i j} \\ \hat{Q}_{22}^{i j} & \bar{Q}_{22}^{i j}\end{array}\right], Q_{f 22}^{i j}=\left[\begin{array}{cc}\bar{Q}_{f 22}^{i j} & \tilde{Q}_{f 22}^{i j} \\ \bar{Q}_{f 22}^{i j} & \hat{Q}_{f 22}^{i j}\end{array}\right]$, $\left(j=1, \ldots, \gamma_{1}\right), \quad Q_{22}^{i j}=\left[\begin{array}{l}\hat{Q}_{22}^{i j} \\ \bar{Q}_{22}^{i j}\end{array}\right], \quad Q_{f 22}^{i j}=\left[\begin{array}{l}\hat{Q}_{f 22}^{i j} \\ \bar{Q}_{f 22}^{i j}\end{array}\right], \quad\left(\mathrm{j}=\gamma_{1}+1, \ldots, \quad \gamma\right) \quad$ with $\quad \hat{x}_{2}^{i} \in R$, $\bar{x}_{2}^{i} \in R^{\left(v_{i}-1\right) \times 1}, \quad \hat{p}_{2}^{i} \in R, \quad \hat{B}_{2}^{i} \in R^{1 \times m}, \quad \hat{B}_{2}^{i} \in R^{1 \times m}, \quad \bar{B}_{2}^{i} \in R^{\left(v_{i}-1\right) \times m}, \quad \hat{Q}_{21}^{i} \in R^{1 \times \rho}$, $\bar{Q}_{21}^{i} \in R^{\left(v_{i}-1\right) \times \rho}, \quad \hat{Q}_{22}^{i j} \in R, \quad \breve{Q}_{22}^{i j}=\left(\tilde{Q}_{22}^{j i}\right)^{T} \in R^{1 \times\left(v_{j}-1\right)}, \quad \tilde{Q}_{22}^{i j}=\left(\breve{Q}_{22}^{j i}\right)^{T} \in R^{\left(v_{i}-1\right) \times 1}$, $\bar{Q}_{22}^{i j}=\left(\bar{Q}_{22}^{j i}\right)^{T} \in R^{\left(v_{i}-1\right) \times\left(v_{j}-1\right)}, \quad \hat{Q}_{f 21}^{i} \in R^{1 \times \rho}, \quad \bar{Q}_{f 21}^{i} \in R^{\left(v_{i}-1\right) \times \rho}, \quad \hat{Q}_{f 22}^{i j} \in R$, $\tilde{Q}_{f 22}^{i j} \in R^{\left(v_{i}-1\right) \times 1}, \tilde{Q}_{f 22}^{i j} \in R^{\left(v_{i}-1\right) \times 1}, \bar{Q}_{f 22}^{i j}=\left(\bar{Q}_{f 22}^{j i}\right)^{T} \in R^{\left(v_{i}-1\right) \times\left(v_{j}-1\right)}$.

In the Weierstrass form, (2) can be expressed as

$$
\begin{aligned}
& \dot{x}_{1}(t)=A_{1} x_{1}(t)+B_{1} u(t), x_{1}(0)=x_{10} \\
& N_{i} \dot{x}_{2}^{i}(t)=x_{2}^{i}(t)+B_{2}^{i} u(t), N_{i} x_{2}^{i}(0)=N_{i} x_{20}^{i}
\end{aligned}
$$

with $i=1, \ldots, \gamma$. The solution to (4) is given by

$$
x_{2}^{i}(t)=-\sum_{j=1}^{v_{i}-1} \delta^{(j-1)}(t) N_{i}^{j} x_{2}^{i}(0)-\sum_{j=0}^{v_{i}-1} N_{i}^{j} B_{2}^{i} u^{(j)}
$$

with $\mathrm{I}=1, \ldots, \gamma$. (3)-(4) is impulse controllable (controllable at $\infty$ ) if and only if the rows of $\mathrm{B}_{2}$ corresponding to the bottom rows of the nontrivial Jordan blocks $\left(\mathrm{v}_{\mathrm{i}}>1\right)$ of $\mathrm{N}$ are linearly independent [9]. The LQR problem for impulse controllable singular systems have been solved, so we assume that (3)-(4) is not impulse controllable. Without loss of generality, the following assumption is made.

Assumption 1: $\hat{B}_{2}^{1}, \ldots, \hat{B}_{2}^{\gamma_{1}}$ are linearly independent and the matrix composed of the rows of $B_{2}$ corresponding to the bottom rows of the Jordan blocks $1, \ldots, \gamma_{2}$ has rank of $\gamma_{1}$.

Assumption 1 means that only impulsive modes corresponding to the nontrivial Jordon blocks $1, \ldots, \gamma_{1}$ are controllable and impulsive modes for the Jordon blocks $\gamma_{1}+1, \ldots, \gamma_{2}$ are uncontrollable. With this assumption, any impulses caused by the initial conditions can be removed by a feedback for subsystems corresponding to the Jordon blocks $1, \ldots, \gamma_{1}$.

The following assumptions are made so that solutions to subsystems corresponding to the Jordon blocks $\gamma_{1}+1, \ldots, \gamma_{2}$ are impulse-free.

Assumption 2: $N_{i} B_{2}^{i}=0$ for $i=\gamma_{1}+1, \ldots, \gamma_{2}$.

Assumption 3: $N_{i} x_{20}^{i}=0$ for $i=\gamma_{1}+1, \ldots, \gamma_{2}$.
The following assumptions are sufficient for solutions to the costate equation of the LQR problem to be impulse-free.

Assumption 4: $N_{i}^{T} Q_{21}^{i}=0$ for $i=\gamma_{1}+1, \ldots, \gamma_{2}, \quad N_{i}^{T} Q_{22}^{i j}=0$ for $i=\gamma_{1}+1, \ldots, \gamma_{2}, j=1, \ldots, \gamma$.

Assumption 5: $N_{i}^{T} Q_{f 21}^{i}=0$ for $i=\gamma_{1}+1, \ldots, \gamma_{2}, N_{i}^{T} Q_{f 22}^{i j} N_{j}=0$ for $i=\gamma_{1}+1, \ldots, \gamma_{2}, j=1, \ldots, \gamma$

In addition, in order to make the cost function finite for the case of an infinite horizon, the following assumption is necessary.

Assumption 6: The finite modes of (2) are stabilizable, that is, $\left(A_{1}, B_{1}\right)$ is stabilizable.

\section{Necessary Conditions}

The necessary conditions for $J$ to be minimized are

$$
\begin{aligned}
& E \dot{x}(t)=\partial H / \partial p \\
& E^{T} \dot{p}(t)=-\partial H / \partial x \\
& 0=\partial H / \partial u
\end{aligned}
$$

with $H=x^{T} Q x+u^{T} R u+p^{T}(A x+B u)$ and the boundary conditions $E x(0)=E x_{0}$ and $E^{T} p\left(t_{f}\right)=E^{T} Q_{f} E x\left(t_{f}\right)$, which, in the Weierstrass form, can be written as

$$
\begin{aligned}
& x_{1}(0)=x_{10} \\
& N_{i} x_{2}^{i}(0)=N_{i} x_{20}^{i} \\
& p_{1}\left(t_{f}\right)=Q_{f 11} x_{1}\left(t_{f}\right)+\sum_{j=1}^{\gamma}\left(N_{j}^{T} Q_{f 21}^{j}\right)^{T} x_{2}^{j}\left(t_{f}\right) \\
& N_{i}^{T} p_{2}^{i}\left(t_{f}\right)=N_{i}^{T} Q_{f 21}^{i} x_{1}\left(t_{f}\right)+\sum_{j=1}^{\gamma} N_{i}^{T} Q_{f 22}^{i j} N_{j} x_{2}^{j}\left(t_{f}\right)
\end{aligned}
$$

where $i=1, \ldots, \gamma$. It follows from (6)-(8) that

$$
\begin{aligned}
& E \dot{x}(t)=A x(t)+B u(t) \\
& E^{T} \dot{p}(t)=-Q x(t)-A^{T} p(t) \\
& 0=B^{T} p(t)+R u(t)
\end{aligned}
$$

which, in the Weierstrass form, can be expressed as

$$
\begin{aligned}
& \dot{x}_{1}(t)=A_{1} x_{1}(t)+B_{1} u(t) \\
& N_{i} \dot{x}_{2}^{i}(t)=x_{2}^{i}(t)+B_{2}^{i} u(t) \\
& \dot{p}_{1}(t)=-Q_{11} x_{1}(t)-\sum_{j=1}^{\gamma}\left(Q_{21}^{j}\right)^{T} x_{2}^{j}(t)-A_{1}^{T} p_{1}(t) \\
& N_{i}^{T} \dot{p}_{2}^{i}(t)=-Q_{21}^{i} x_{1}(t)-\sum_{j=1}^{\gamma} Q_{22}^{i j} x_{2}^{j}(t)-p_{2}^{i}(t) \\
& 0=B_{1}^{T} p_{1}(t)+\sum_{j=1}^{\gamma}\left(B_{2}^{j}\right)^{T} p_{2}^{j}(t)+R u(t)
\end{aligned}
$$

where $i=1, \ldots, \gamma$.

\section{Solutions of the LQR Problem}

This section is devoted to finding a solution to the two-point boundary value problem described by (16)-(20) with the boundary conditions (9)-(12). Before doing this, first let us examine the implication of Assumptions 2-3.

With the special structure of $N_{i}$ in mind, the following lemma can be verified using Assumption 2.

Lemma 1: If Assumption 2 is satisfied, then $\left(B_{2}^{i}\right)_{j}=0$ for $i=\gamma_{1}+1, \ldots, \gamma_{2}$, $i=\gamma_{1}+1, \ldots, \gamma_{2}$, where $\left(B_{2}^{i}\right)_{j}$ is the $\mathrm{j}$-th row of $B_{2}^{i}$. 
Citation: Liu X, Jia Y (2016) Linear Quadratic Regulator for Impulse Uncontrollable Singular Systems. J Electr Electron Syst 5: 207. doi:10.4172/23320796.1000207

Proof. A simple calculation shows that

$$
N_{i} B_{2}^{i}=\left[\begin{array}{c}
\left(B_{2}^{i}\right)_{2} \\
\vdots \\
\left(B_{2}^{i}\right)_{v_{i}} \\
0
\end{array}\right]=\left[\begin{array}{c}
0 \\
\vdots \\
0 \\
0
\end{array}\right]
$$

for $i=\gamma_{1}+1, \ldots, \gamma_{2}$, from which the conclusion of the lemma can be directly drawn.

Assumptions 2-3 are sufficient for solutions of the subsystems corresponding to the Jordon blocks $\gamma_{1}+1, \ldots, \gamma_{2}$ to be impulse-free, which will be proved in the following lemma.

Lemma 2: If Assumptions 2 and 3 are satisfied, then $\bar{x}_{2}^{i}(t)=0$ for $i=\gamma_{1}+1, \ldots, \gamma_{2}$.

Proof. Note that Assumption 3 implies $N_{i} x_{2}^{i}(0)=N_{i} x_{20}^{i}=0$ for $i=\gamma_{1}+1, \ldots, \gamma_{2}$. Applying this and Assumptions 2 to (5) gives

$$
x_{2}^{i}(t)=\left[\begin{array}{l}
\hat{x}_{2}^{i}(t) \\
\bar{x}_{2}^{i}(t)
\end{array}\right]=-\sum_{j=1}^{v_{i}-1} \delta^{(j-1)} N_{i}^{j-1} N_{i} x_{2}^{i}(0)-B_{2}^{i} u(t)-\sum_{j=1}^{v_{j}-1} N_{i}^{j-1}\left(N_{i} B_{2}^{i}\right) u^{(j)}(t)=-B_{2}^{i} u(t)
$$

which, according to Lemma 1 , means that $\bar{x}_{2}^{i}(t)=0$ for $i=\gamma_{1}+1, \ldots, \gamma_{2}$.

Similar to the proof of Lemma 1, it is straightforward to prove the following lemma.

Lemma 3: If Assumption 4 is satisfied, then $\left(Q_{21}^{i}\right)_{k}=0$ for $k=1, \ldots, v_{i}-1, i=\gamma_{1}+1, \ldots, \gamma_{2}$, and $\left(Q_{22}^{i j}\right)_{k}=0$ for $\mathrm{k}=1 k=1, \ldots, v_{i}-1$, $i=\gamma_{1}+1, \ldots, \gamma_{2}, j=1, \ldots, \gamma$, where $\left(Q_{21}^{i}\right)_{k}$ and $\left(Q_{22}^{i j}\right)_{k}$ are the k-th rows of $Q_{21}^{i}$ and $Q_{22}^{i j}$, respectively.

Proof. The conclusion can be drawn from the relations.

$$
\begin{gathered}
N_{i}^{T} Q_{21}^{i}=\left[\begin{array}{c}
0 \\
\left(Q_{21}^{i}\right)_{1} \\
\vdots \\
\left(Q_{21}^{i}\right)_{v_{i}-1}
\end{array}\right]=\left[\begin{array}{c}
0 \\
0 \\
\vdots \\
0
\end{array}\right] \\
N_{i}^{T} Q_{22}^{i j}=\left[\begin{array}{c}
0 \\
\left(Q_{22}^{i j}\right)_{1} \\
\vdots \\
\left(Q_{22}^{i j}\right)_{v_{i}-1}
\end{array}\right]=\left[\begin{array}{c}
0 \\
0 \\
\vdots \\
0
\end{array}\right] .
\end{gathered}
$$

The following lemma indicates that the final values for $\bar{p}_{2}^{i}$ are zeros for $i=\gamma_{1}+1, \ldots, \gamma_{2}$ if Assumption 5 is satisfied.

Lemma 4: If Assumption 5 is satisfied, then $\bar{p}_{2}^{i}\left(t_{f}\right)=0$ for $i=\gamma_{1}+1, \ldots, \gamma_{2}$.

Proof. Due to Assumption 5, (12) can be rewritten as

$$
\left[\begin{array}{c}
0 \\
\bar{p}_{2}^{i}\left(t_{f}\right)
\end{array}\right]=N_{i}^{T} Q_{f 21}^{i} x_{1}\left(t_{f}\right)+\sum_{j=1}^{\gamma} N_{i}^{T} Q_{f 22}^{i j} N_{j} x_{2}^{j}\left(t_{f}\right)=\left[\begin{array}{l}
0 \\
0
\end{array}\right]
$$

The following lemma shows that solutions to the costate subsystems corresponding to the Jordon blocks $\gamma_{1}+1, \ldots, \gamma_{2}$ are impulse-free if Assumptions 4-5 are satisfied.

Lemma 5: If Assumptions 4-5 are satisfied, then $\bar{p}_{2}^{i}(t)=0$ for $i=\gamma_{1}+1, \ldots, \gamma_{2}$.

Proof. It can be proved that solutions to (19) is given by

$$
p_{2}^{i}(t)=-\sum_{k=1}^{v_{i}-1} \delta^{(k-1)}\left(t-t_{f}\right)\left(-N_{i}^{T}\right)^{k-1}\left(-N_{i}^{T}\right) p_{2}^{i}\left(t_{f}\right)
$$

$$
-\sum_{k=0}^{v_{i}-1}\left(-N_{i}^{T}\right)^{k}\left[Q_{21}^{i} x_{1}^{(k)}(t)+\sum_{j=1}^{\gamma} Q_{22}^{i j}\left(x_{2}^{j}\right)^{(k)}(t)\right]
$$

for $i=\gamma_{1}+1, \ldots, \gamma_{2}$. It follows from Lemma 4 that $\left(-N_{i}^{T}\right) p_{2}^{i}\left(t_{f}\right)=0$ for $i=\gamma_{1}+1, \ldots, \gamma_{2}$. As a result, with Assumption $4, p_{2}^{i}(t)$ can be simplified as

$$
\left[\begin{array}{c}
\bar{p}_{2}^{i} \\
\hat{p}_{2}^{i}
\end{array}\right]=-\sum_{k=0}^{v_{i}-1}\left(-N_{i}^{T}\right)^{k}\left[Q_{21}^{i} x_{1}^{(k)}+\sum_{j=1}^{\gamma} Q_{22}^{i j}\left(x_{2}^{j}\right)^{(k)}\right]=-Q_{21}^{i} x_{1}-\sum_{j=1}^{\gamma} Q_{22}^{i j} x_{2}^{j}
$$

which, together with Lemma 3, implies that the lemma holds.

A simple manipulation shows that for ${ }^{i=1, \ldots, \gamma_{1}},(17)$ and (19) can be expressed as

$$
\begin{aligned}
& \dot{\bar{x}}_{2}^{i}=A_{11}^{i} \bar{x}_{2}^{i}+A_{1}^{i} \hat{x}_{2}^{i}+\bar{B}_{2}^{i} u \\
& 0=A_{21}^{i} \bar{x}_{2}^{i}+\hat{B}_{2}^{i} u
\end{aligned}
$$

And

$$
\begin{aligned}
& 0=-\hat{Q}_{21}^{i} x_{1}-\sum_{j=1}^{\gamma_{1}} \hat{Q}_{22}^{i j} \hat{x}_{2}^{j}-\sum_{j=1}^{\gamma_{1}} \breve{Q}_{22}^{i j} \bar{x}_{2}^{j}-\sum_{j=\gamma_{1}+1}^{\gamma} \hat{Q}_{22}^{i j} x_{2}^{j}-\left(A_{12}^{i}\right)^{T} \bar{p}_{2}^{i} \\
& \bar{p}_{2}^{i}=-\bar{Q}_{21}^{i} x_{1}-\sum_{j=1}^{\gamma_{1}} \tilde{Q}_{22}^{i j} \hat{x}_{2}^{j}-\sum_{j=1}^{\gamma_{1}} \bar{Q}_{22}^{i j} \bar{x}_{2}^{j}-\sum_{j=\gamma_{1}+1}^{\gamma} \bar{Q}_{22}^{i j} x_{2}^{j}-\left(A_{11}^{i}\right)^{T} \bar{p}_{2}^{i}-\left(A_{21}^{i}\right)^{T} \hat{p}_{2}^{i}
\end{aligned}
$$

where $\quad A_{12}^{i}=\left[\begin{array}{llll}1 & 0 & \cdots & 0\end{array}\right]^{T} \in R^{\left(v_{i}-1\right) \times 1}, \quad A_{21}^{i}=\left[\begin{array}{llll}0 & \cdots & 0 & 1\end{array}\right] \in R^{1 \times\left(v_{i}-1\right)}$, $A_{11}^{i}=\left[\begin{array}{cc}0 & I_{v_{i}-2} \\ 0 & 0\end{array}\right]^{T} \in R^{\left(v_{i}-1\right) \times\left(v_{i}-1\right)}$.

It follows from Lemma 3 and Lemma 5 that $N_{i} \dot{x}_{2}^{i}(t)=0$ and $N_{i}^{T} \dot{p}_{2}^{i}(t)=0$ due to $\bar{x}_{2}^{i}(t)=0$ and $\bar{p}_{2}^{i}(t)=0$ for $i=\gamma_{1}+1, \ldots, \gamma_{2}$. As a result, (17) and (19), for $i=\gamma_{1}+1, \ldots, \gamma$, are equivalent to

$$
0=x_{2}^{i}+B_{2}^{i} u
$$

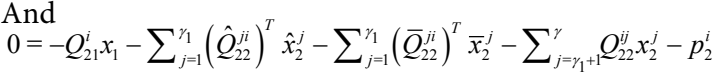

due to $v_{i}=1$ for $i=\gamma_{2}+1, \ldots, \gamma$. (18) and (20) can be described as

$$
\begin{aligned}
& \dot{p}_{1}=-Q_{11} x_{1}-\sum_{j=1}^{\gamma_{1}}\left(\hat{Q}_{21}^{j}\right)^{T} \hat{x}_{2}^{j}-\sum_{j=1}^{\gamma_{1}}\left(\bar{Q}_{21}^{j}\right)^{T} \bar{x}_{2}^{j}-\sum_{j=\gamma_{1}+1}^{\gamma}\left(Q_{21}^{j}\right)^{T} x_{2}^{j}-A_{1}^{T} p_{1} \\
& 0=B_{1}^{T} p_{1}+\sum_{j=1}^{\gamma_{1}}\left(\bar{B}_{2}^{j}\right)^{T} \bar{p}_{2}^{j}+\sum_{j=1}^{\gamma_{1}}\left(\hat{B}_{2}^{j}\right)^{T} \hat{p}_{2}^{j}+\sum_{j=\gamma_{1}+1}^{\gamma}\left(B_{2}^{j}\right)^{T} p_{2}^{j}+R u
\end{aligned}
$$

The initial conditions (10) can be expressed as

$$
\bar{x}_{2}^{i}(0)=\bar{x}_{20}^{i}, i=1, \ldots, \gamma_{1}
$$

Similarly, the final conditions (11) and (12) can be written as

$$
\begin{aligned}
& p_{1}\left(t_{f}\right)=Q_{f 11} x_{1}\left(t_{f}\right)+\sum_{j=1}^{\gamma_{1}}\left(\bar{Q}_{f 21}^{j}\right)^{T} \bar{x}_{2}^{j}\left(t_{f}\right) \\
& \bar{p}_{2}^{i}\left(t_{f}\right)=\bar{Q}_{f 21}^{i} x_{1}\left(t_{f}\right)+\sum_{j=1}^{\gamma_{1}} \bar{Q}_{f 22}^{i j} \bar{x}_{2}^{j}\left(t_{f}\right)
\end{aligned}
$$

for $i=1, \ldots, \gamma_{1}$, where $\bar{x}_{2}^{j}\left(t_{f}\right)=0$ due to $\bar{x}_{2}^{j}(t)=0$ for $j=\gamma_{1}+1, \ldots, \gamma_{2}$ and $N_{j}^{T} Q_{f 21}^{j}=0$ due to $v_{j}=1$ for $j=\gamma_{2}+1, \ldots, \gamma$ are used.

To simplify expressions, define the following matrices $\hat{Q}_{22}=\left[\begin{array}{ll}\tilde{Q}_{1} & \tilde{Q}_{2}\end{array}\right]$,

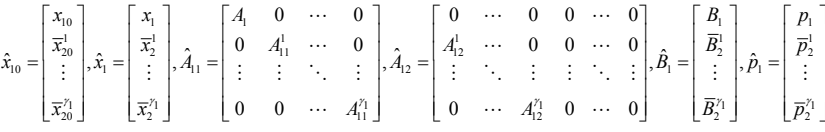

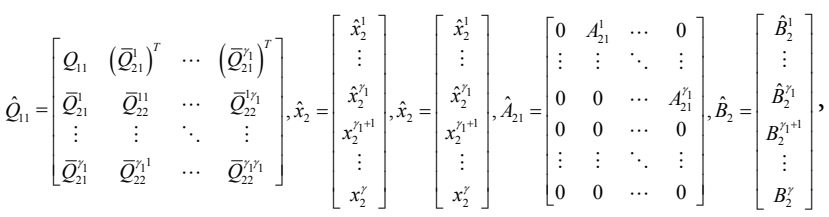




$$
\begin{aligned}
& \hat{A}_{22}=\left[\begin{array}{cccccc}
0 & \cdots & 0 & 0 & \cdots & 0 \\
\vdots & \ddots & \vdots & \vdots & \ddots & \vdots \\
0 & \cdots & 0 & 0 & \cdots & 0 \\
0 & \cdots & 0 & I & \cdots & 0 \\
\vdots & \ddots & \vdots & \vdots & \ddots & \vdots \\
0 & \cdots & 0 & 0 & \cdots & I
\end{array}\right], \hat{Q}_{f 11}=\left[\begin{array}{cccc}
Q_{f 11} & \left(\bar{Q}_{f 21}^{1}\right)^{T} & \cdots & \left(\bar{Q}_{f 12}^{\prime}\right)^{T} \\
\bar{Q}_{f 21}^{1} & \bar{Q}_{f 22}^{11} & \cdots & \bar{Q}_{f 22}^{1,1} \\
\vdots & \vdots & \ddots & \vdots \\
\bar{Q}_{f 21}^{\prime 1} & \bar{Q}_{f 22}^{\prime 11} & \cdots & \bar{Q}_{f 22}^{\gamma \gamma 1}
\end{array}\right],
\end{aligned}
$$

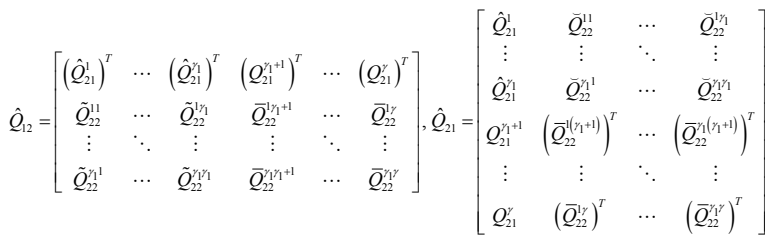

with

$$
\tilde{Q}_{1}=\left[\begin{array}{ccc}
\hat{Q}_{22}^{11} & \cdots & \hat{Q}_{22}^{1 / \gamma_{1}} \\
\vdots & \ddots & \vdots \\
\hat{Q}_{22}^{\gamma_{1} 1} & \cdots & \hat{Q}_{22}^{\gamma_{1} \gamma_{1}} \\
\left(\hat{Q}_{22}^{1\left(\gamma_{1}+1\right)}\right)^{T} & \cdots & \left(\hat{Q}_{22}^{\gamma_{1}\left(\gamma_{1}+1\right)}\right)^{T} \\
\vdots & \ddots & \vdots \\
\left(\hat{Q}_{22}^{1 \gamma}\right)^{T} & \cdots & \left(\hat{Q}_{22}^{\gamma_{1} \gamma}\right)^{T}
\end{array}\right], \tilde{Q}_{2}=\left[\begin{array}{ccc}
\hat{Q}_{22}^{\left(\gamma_{1}+1\right)} & \cdots & \hat{Q}_{22}^{1 \gamma} \\
\vdots & \ddots & \vdots \\
\hat{Q}_{22}^{\gamma_{1}\left(\gamma_{1}+1\right)} & \cdots & \hat{Q}_{22}^{\gamma_{1} \gamma} \\
Q_{22}^{\left(\gamma_{1}+1\right)\left(\gamma_{1}+1\right)} & \cdots & Q_{22}^{\left(\gamma_{1}+1\right) \gamma} \\
\vdots & \ddots & \vdots \\
Q_{22}^{\gamma\left(\gamma_{1}+1\right)} & \cdots & Q_{22}^{\gamma \gamma}
\end{array}\right],
$$

It is straightforward to prove that $\hat{Q}_{12}=\hat{Q}_{21}^{T}$.

Combining (16) and (21) yields

$\dot{\hat{x}}_{1}(t)=\hat{A}_{11} \hat{x}_{1}(t)+\hat{A}_{12} \hat{x}_{2}(t)+\hat{B}_{1} u$

and combining (22) and (25) produces

$0=\hat{A}_{21} \hat{x}_{1}(t)+\hat{A}_{22} \hat{x}_{2}(t)+\hat{B}_{2} u$

Similarly, (27), and (24) can be combined to get

$\dot{\hat{p}}_{1}(t)=-\hat{Q}_{11} \hat{x}_{1}(t)-\hat{Q}_{12} \hat{x}_{2}(t)-\hat{A}_{11}^{T} \hat{p}_{1}(t)-\hat{A}_{21}^{T} \hat{p}_{2}(t)$

and (23) and (26) can be put together to obtain

$0=-\hat{Q}_{21} \hat{x}_{1}(t)-\hat{Q}_{22} \hat{x}_{2}(t)-\hat{A}_{12}^{T} \hat{p}_{1}(t)-\hat{A}_{22}^{T} \hat{p}_{2}(t)$

In addition, (28) can be rewritten as

$0=\hat{B}_{1}^{T} \hat{p}_{1}(t)+\hat{B}_{2}^{T} \hat{p}_{2}(t)+R u(t)$

The initial conditions (9) and (29) can be written as

$\hat{x}_{1}(0)=\hat{x}_{10}$

The final conditions (30) and (31) can be described as $\hat{p}_{1}\left(t_{f}\right)=\hat{Q}_{f 11} \hat{x}_{1}\left(t_{f}\right)$

It will prove convenient to define the following matrices

$$
\bar{S}=\left[\begin{array}{lll}
\hat{A}_{21}^{T} & \hat{Q}_{12} & 0
\end{array}\right], \bar{B}=\left[\begin{array}{lll}
0 & \hat{A}_{12} & \hat{B}_{1}
\end{array}\right], \bar{R}=\left[\begin{array}{ccc}
0 & \hat{A}_{22} & \hat{B}_{2} \\
\hat{A}_{22}^{T} & \hat{Q}_{22} & 0 \\
\hat{B}_{2}^{T} & 0 & R
\end{array}\right]
$$$$
\text { with } \hat{A}_{22}=\left[\begin{array}{cc}
0 & 0 \\
0 & I
\end{array}\right], \hat{B}_{2}=\left[\begin{array}{c}
\hat{b}_{2}^{1} \\
\hat{b}_{2}^{2}
\end{array}\right], \hat{b}_{2}^{1}=\left[\begin{array}{c}
\hat{B}_{2}^{1} \\
\vdots \\
\hat{B}_{2}^{\gamma_{1}}
\end{array}\right]
$$

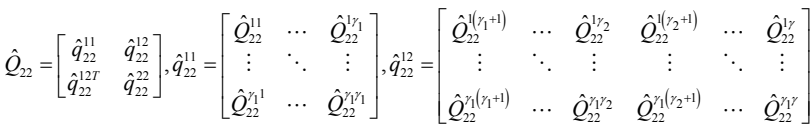

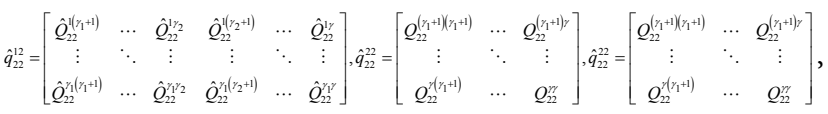

Lemma 6: If Assumption 1 is satisfied, then the matrix $\hat{b}_{2}^{1}$ has full row rank.

Proof. This lemma can be easily verified by applying Assumption 1 .

Assumption 7: $\tilde{R}=R-\hat{b}_{2}^{2 T}\left[-\hat{q}_{22}^{22}+\hat{q}_{22}^{12 T}\left(\hat{q}_{22}^{11}\right)^{-1} \hat{q}_{22}^{12}\right] \hat{b}_{2}^{2}>0$ and $\hat{q}_{22}^{11}>0$.

Lemma 7: If Assumptions 1 and 7 are satisfied, then $\bar{R}$ is invertible.

Proof. Note that

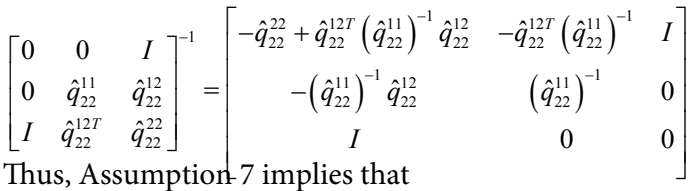

$$
\left[\begin{array}{cccc}
0 & 0 & I & \hat{b}_{2}^{2} \\
0 & \hat{q}_{22}^{11} & \hat{q}_{22}^{12} & 0 \\
I & \hat{q}_{22}^{12 T} & \hat{q}_{22}^{22} & 0 \\
\hat{b}_{2}^{2 T} & 0 & 0 & R
\end{array}\right]^{-1}=R-\left[\begin{array}{c}
\hat{b}_{2}^{2} \\
0 \\
0
\end{array}\right]^{T}\left[\begin{array}{ccc}
0 & 0 & I \\
0 & \hat{q}_{22}^{11} & \hat{q}_{22}^{12} \\
I & \hat{q}_{22}^{12 T} & \hat{q}_{22}^{22}
\end{array}\right]^{-1}\left[\begin{array}{c}
\hat{b}_{2}^{2} \\
0 \\
0
\end{array}\right]=\tilde{R}
$$

is invertible. Therefore, according to Lemma $6, \bar{R}$ is invertible.

Therefore, $\hat{x}_{2}(t), \hat{p}_{2}(t)$, and $\mathrm{u}(\mathrm{t})$ can be uniquely determined from (33), (35), and (36) as

$$
\left[\begin{array}{c}
\hat{p}_{2}(t) \\
\hat{x}_{2}(t) \\
u(t)
\end{array}\right]=-\bar{R}^{-1} \bar{S}^{T} \hat{x}_{1}(t)-\bar{R}^{-1} \bar{B}^{T} \hat{p}_{1}(t)
$$

This can then be substituted back into (32) and (34), which results in a usual two-point boundary value problem of linear-quadratic optimal control with $\hat{x}_{1}(t)$ and $\hat{p}_{1}(t)$ as the state and costate instead of $\mathrm{x}(\mathrm{t})$ and $\mathrm{p}(\mathrm{t})$

(32) and (34) becomes

$$
\left[\begin{array}{l}
\dot{\hat{x}}_{1}(t) \\
\hat{\hat{p}}_{1}(t)
\end{array}\right]=\left[\begin{array}{cc}
\hat{A} & -\hat{R} \\
-\hat{Q} & -\hat{A}^{T}
\end{array}\right]\left[\begin{array}{l}
\hat{x}_{1}(t) \\
\hat{p}_{1}(t)
\end{array}\right]
$$

with the boundary conditions of (37) and (38) where $\hat{A}=\hat{A}_{11}-\bar{B} \bar{R}^{-1} \bar{S}^{T}$, $\hat{R}=\bar{B} \bar{R}^{-1} \bar{B}^{T}$, and $\hat{Q}=\hat{Q}_{11}-\overline{S R}^{-1} \bar{S}^{T}$.

The solutions to the two-point boundary value problem (40) can be solved by computing its back-time state transition matrix

$$
\left[\begin{array}{l}
\hat{x}_{1}(t) \\
\hat{p}_{1}(t)
\end{array}\right]=\left[\begin{array}{ll}
\Omega_{11}\left(t, t_{f}\right) & \Omega_{12}\left(t, t_{f}\right) \\
\Omega_{21}\left(t, t_{f}\right) & \Omega_{22}\left(t, t_{f}\right)
\end{array}\right]\left[\begin{array}{l}
\hat{x}_{1}\left(t_{f}\right) \\
\hat{p}_{1}\left(t_{f}\right)
\end{array}\right]
$$

By following the approach proposed by Bender and Laub [5], the optimal feedback gain can be determined by solving the equation

$$
U(t)=K(t) X(t)
$$

for $K(t)$ with $X(t)=\left[\begin{array}{l}\hat{X}_{1}(t) \\ \hat{X}_{2}(t)\end{array}\right]$. The general solutions is

$$
K(t)=U(t) X^{+}(t)+Y(t)\left[I-X(t) X^{+}(t)\right]
$$

where $Y(t) \in R^{m \times n}$ is an arbitrary real matrix and $\mathrm{U}$ can be determined from

$$
\left[\begin{array}{c}
\hat{P}_{2} \\
\hat{X}_{2} \\
U
\end{array}\right]=-\bar{R}^{-1}\left[\begin{array}{cc}
\hat{A}_{21} & 0 \\
\hat{Q}_{21} & \hat{A}_{12}^{T} \\
0 & \hat{B}_{1}^{T}
\end{array}\right]\left[\begin{array}{l}
I \\
P
\end{array}\right] \hat{X}_{1}
$$


Citation: Liu X, Jia Y (2016) Linear Quadratic Regulator for Impulse Uncontrollable Singular Systems. J Electr Electron Syst 5: 207. doi:10.4172/23320796.1000207

Page 5 of 5

With $\mathrm{P}$ being the solution of the following Riccati equation

$-\dot{P}=P \hat{A}+\hat{A}^{T} P+\hat{Q}-P \hat{R}^{-1} P, P\left(t_{f}\right)=\hat{Q}_{f 11}$

The main results can be summarized by the following theorem.

Theorem 1. Assume that the singular system is in the Weierstrass form. If Assumptions 1-7 are satisfied, then

(1) there exists a unique, impulse-free solution of the necessary conditions (16)-(20), together with the boundary conditions (9)-(12), which can be found from (39) and (40);

(2) the optimal feedback gain can be determined by (43);

(3) the optimal cost J from (1) is given by $J=\frac{1}{2} \hat{x}_{10}^{T} P(0) \hat{x}_{10}$

\section{Examples}

Example 1. Consider a simple example of a planar manipulator constrained so that the end effector can only move along a straight line. The equations of motion [10] is given by

$$
\begin{aligned}
& \ddot{q}_{1}=u_{1}+f_{1} \\
& \ddot{q}_{2}=u_{2}+f_{2}
\end{aligned}
$$

with a constraint equation of $q_{1}+2 q_{2}=1$.

Thus, the forces of constraint are $f_{1}=\lambda$ and $f_{2}=2 \lambda$. Set $x_{1}=2 q_{1}-q_{2}, x_{2}=2 \dot{q}_{1}-\dot{q}_{2}, x_{3}=5 \lambda, x_{4}=\dot{q}_{1}+2 \dot{q}_{2}, x_{5}=q_{1}+2 q_{2}-1$. Then, the manipulator can be expressed as the form of (3)-(4) with $A_{1}=\left[\begin{array}{ll}0 & 1 \\ 0 & 0\end{array}\right], \quad B_{1}=\left[\begin{array}{cc}0 & 0 \\ 2 & -1\end{array}\right], \quad N_{1}=\left[\begin{array}{lll}0 & 1 & 0 \\ 0 & 0 & 1 \\ 0 & 0 & 0\end{array}\right], \quad B_{2}^{1}=\left[\begin{array}{cc}1 & 2 \\ 0 & 0 \\ 0 & 0\end{array}\right]$. This system is not impulse controllable with $\gamma_{1}=0, \gamma=\gamma_{2}=1$. It can be verified that Assumptions 1-7 are satisfied with initial conditions $q_{10}+2 q_{20}-1=0$, $\dot{q}_{10}+2 \dot{q}_{20}=0$, and matrices $Q_{11}=\left[\begin{array}{ll}1 & 0 \\ 0 & 1\end{array}\right], \quad Q_{12}=\left[\begin{array}{lll}0 & 0 & 5 \\ 0 & 0 & 6\end{array}\right]$, $Q_{22}=\left[\begin{array}{lll}0 & 0 & 0 \\ 0 & 0 & 0 \\ 0 & 0 & 3\end{array}\right], Q_{f 22}=Q_{22}, Q_{21}=Q_{12}^{T}$, and $Q_{f 21}=Q_{f 21}^{T}=Q_{12}$. It is simple to verify the following: $\hat{B}_{1}=B_{1}, \hat{B}_{2}=B_{2}^{1}, \hat{Q}_{21}=Q_{21}, \hat{Q}_{22}=Q_{22}, \hat{Q}_{22}=Q_{22}$,

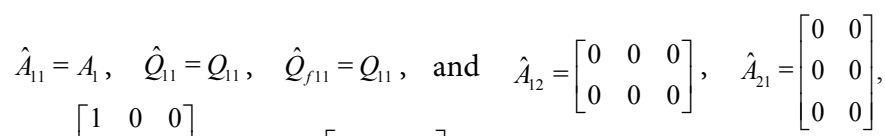
$\hat{A}_{22}=\left[\begin{array}{lll}1 & 0 & 0 \\ 0 & 1 & 0 \\ 0 & 0 & 1\end{array}\right]$. Set $P=\left[\begin{array}{ll}p_{11} & p_{21} \\ p_{21} & p_{22}\end{array}\right]$. Then, the Riccati equation

for the case of $t_{f}=\infty$ has a unique positive definite solution of $P=\left[\begin{array}{cc}\frac{1}{5} \sqrt{10 \sqrt{5}+25} & \frac{1}{5} \sqrt{5} \\ \frac{1}{5} \sqrt{5} & \frac{1}{5} \sqrt{2 \sqrt{5}+5}\end{array}\right]$. It follows from (44) that $\hat{X}_{2}(t)=0$ and $U(t)=K_{1} \hat{X}_{1}(t)$ with $K_{1}=\left[\begin{array}{cc}-\frac{2}{5} \sqrt{5} & -\frac{2}{5} \sqrt{2 \sqrt{5}+5} \\ \frac{1}{5} \sqrt{5} & \frac{1}{5} \sqrt{2 \sqrt{5}+5}\end{array}\right]$. According to (42), the feedback $K=\left[\begin{array}{ll}K_{1} & K_{2}\end{array}\right]$ can be found with $\mathrm{K}_{2}$ being arbitrary.

\section{Conclusion}

In this paper, the linear quadratic regulator problem has been solved for a linear time-invariant singular systems which may not be impulse controllable. The impulse controllable and uncontrollable subsystems have been separated by transforming the singular system into the Weierstrass form. The sufficient conditions have been proposed so that the impulse uncontrollable subsystems and their corresponding costate subsystems admit a unique impulse-free solution. The necessary conditions for the minimization of the quadratic cost function have been converted to the Riccati equation.

\section{References}

1. Pandolfi L (1981) On the regulator problem for linear degenerate contro systems. Journal of Optimization Theory and Applications 33: 241-254.

2. Cobb DJ (1983) Descriptor variable systems and optimal state regulation. IEEE Transactions on Automatic Control 28: 601-611.

3. Lewis FL (1985) On singular optimal control for singular systems. Proceedings of IEEE Conference, Decision and Control, pp: 266-272.

4. Lovass-Nagy V, Schilling R, Yan HC (1986) A note on optimal control of generalized state-space (descriptor) systems. International Journal of Control 44: 613-624.

5. Bender DJ, Laub AJ (1987) The linear-quadratic optimal regulator for descriptor systems. IEEE Transactions on Automatic Control 32: 672-688.

6. Wang YY, Frank PM, Clements DJ (1993) The robustness properties of the linear quadratic regulators for singular systems. IEEE Transactions on Automatic Control 38: 96-100.

7. Kunkel P, Mehrmann V (1997) The linear quadratic control problem for linear descriptor systems with variable coefficients. Mathematics of Control, Signals and Systems 10: 247-264.

8. Katayama T, Minamino K (1992) Linear quadratic regulator and spectra factorization for continuous-time descriptor systems. Proceedings of IEEE Conference, Decision and Control, Tucson, Arizona pp: 967-972.

9. Zhu J, Ma S, Cheng ZL (2002) Singular LQ problem for nonregular descriptor systems. IEEE Transactions on Automatic Control 47: 1128-1133.

10. McClamroch NH, Wang D (1988) Feedback stabilization and tracking of constrained robots. IEEE Transactions on Automatic Control 33:419-426.

11. Verghese GC (1978) Infinite-frequency behavior in generalized dynamica systems. Ph D Thesis, Department of Electrical Engineering, Stanford University.

12. Verghese GC, Levy BC, Kailath $T$ (1981) A generalized state-space for singular systems. IEEE Transactions on Automatic Control, 26: 811-831. 